\title{
Structure of a turbulent crossbar near-wake studied by means of lattice Boltzmann simulation
}

\author{
Lyazid Djenidi* \\ School of Engineering, The University of Newcastle, Newcastle 2308 NSW, Australia
}

(Received 1 November 2007; published 18 March 2008)

\begin{abstract}
The turbulent near-wake of a crossbar is investigated numerically with the lattice Boltzmann method (LBM). The crossbar is made up of two perpendicular square bars arranged in a biplane configuration and is included in the computational domain. The Reynolds number based on a bar diameter is about 1600 . The numerical results are first tested against results of both particle image velocimetry (PIV) and laser Doppler velocimetry (LDV). The LBM data compare well with the PIV and LDV data. In particular, the LBM reproduces the generation of vortical structures at the crossbar as observed in the PIV data. The numerical results reveal the presence of intermittent lateral motions along the span of the two bars, yielding fingerlike structures. It is argued that these motions contribute to the formation of streamwise vortical structures just behind the crossbar. These streamwise structures interlace with lateral structures also generated at the crossbar. The region over which this activity takes place is about four diameters. Within this region, the turbulent kinetic energy at the crossbar centerline increases and reaches a maximum at a distance of about three diameters. As the downstream distance increases, the individual wakes merge to form a single wake with features, for $x / D \geq 20$, similar to those observed in grid-generated turbulence.
\end{abstract}

DOI: 10.1103/PhysRevE.77.036310

PACS number(s): 47.27.wb, 47.27.E-, 47.27.De

\section{INTRODUCTION}

Despite the large amount of work on homogeneous isotropic turbulence (HIT), there are still not clear conclusions on issues such as the effects of the initial conditions on the decay of turbulence (the initial conditions here are defined by the grid geometry) and its decay. For example, in gridgenerated turbulence, where turbulence is produced by a fluid flowing uniformly through a grid made of bars, it is commonly accepted that the turbulence kinetic energy decays following a power law, $q \sim x^{n} \quad\left(q=\left\langle u^{2}\right\rangle+\left\langle u^{2}\right\rangle+\left\langle u^{2}\right\rangle\right.$ is twice the turbulent kinetic energy, $x$ is the streamwise distance behind the grid, $n$ is constant, and $u, v$, and $w$ are the velocity components in the streamwise and the two transverse directions). However, while HIT theory predicts that $n=-1$ for an infinite Reynolds number $R_{\lambda}\left(\{=\}\left\langle u^{2}\right\rangle \lambda / \nu, \lambda\right.$ is Taylor's microscale) there is no consensus as to what should be $n$ for finite values of $R_{\lambda}$. In addition, George [1] and George et al. [2] argued that the decay of turbulence is not universal and may be a function of the initial conditions. More recently, a study of a grid-generated turbulence [3-5] showed that initial conditions (i.e., the conditions the grid geometry imposes on the flow) have a persistent impact on the large scale organization of the turbulence. On the other hand, the small scale motion appeared to be less sensitive to the grid geometry. This was seen by using a secondary contraction mounted just behind the grid [6], which improved the isotropy of the large scale motion but left almost unchanged the small scale isotropy [5]. In that context, it is interesting to determine the source of the anisotropy introduced in the flow and how it evolves with the decay of turbulence. The main issues are then to understand the mechanism(s) of the production of turbulence, the redistribution of the turbulent kinetic energy among its components, and how

\footnotetext{
*Lyazid.djenidi@newcastle.edu.au
}

the grid geometry may impact on the decay of turbulence. Since turbulence is produced at large scales, it is then natural to investigate the generation of large scale structures, which, through their interactions, distribute the energy among the turbulent energy components through pressure and nonlinear inertial forces.

The present paper reports on a numerical simulation of a turbulent wake behind a crossbar made of two square cylinders in a biplane configuration. The motivation for studying such a flow stems from the desire to improve the general understanding of the role of the initial conditions on the decay of a grid-generated turbulence. Indeed, one may argue that the crossbar arrangement can be seen as the "unit element" of a grid made of perpendicular bars. This arrangement allows us to study the crossbar wake alone (its generation and decay), which would be difficult in the actual gridgenerated turbulence where the wakes of the "unit elements" interact quickly behind the grid. The emphasis of the work is on the near-field region of the crossbar wake, where the individual wakes of each bar interact strongly. While a large body of work on wakes behind cylinders (single cylinder, cylinder pair, or rows of cylinders) can be found in the literature, not many studies of a crossbar wake, in particular with the aim to investigate it in relation to grid-generated turbulence, are available. Osaka et al. [7,8], for instance, investigated the structure of turbulence behind a crossbar (inplane configuration) with the view to compare the development of the wake with that of a two-dimensional wake behind a cylinder. In the present study, the emphasis is on the generation and development of the large scale vortical structures, whose interaction is the source of turbulence energy production.

\section{NUMERICAL PROCEDURE}

\section{A. The lattice Boltzmann method}

The turbulent wake of the crossbar is simulated using the lattice Boltzmann method. The LBM is based on kinetic 
theory. Rather than solving the governing fluid equations (Navier-Stokes equations), the LBM solves the Boltzmann equation on a lattice. The basic idea of the LBM is to construct a simplified kinetic model that incorporates the essential physics of microscopic average properties, which obey the desired (macroscopic) Navier-Stokes equations [9]. With a sufficient amount of symmetry of the lattice, the LBM implicitly solves these latter equations with second-order accuracy. For the present calculations, each computational node consists of a three dimensional lattice composed of 18 moving particles and a rest particle (lattice model D3Q19). The spacings in the three directions between each node are noted $\Delta x, \Delta y$, and $\Delta z$.

The Boltzmann equation is discretized on that lattice and results in the lattice Boltzmann equation, which governs the time and space variations of the single-particle distribution $f_{i}(\boldsymbol{x}, t)$ at the lattice site $\boldsymbol{x}$ :

$$
f_{i}\left(\boldsymbol{x}+\boldsymbol{e}_{i} \Delta t, t+\Delta t\right)-f_{i}(\boldsymbol{x}, t)=-\frac{1}{\tau}\left[f_{i}(\boldsymbol{x}, t)-f_{i}^{e q}(\boldsymbol{x}, t)\right],
$$

with $i=0,1, \ldots, 18, \tau$ is the single relaxation time, $\Delta t$ the time step, $\boldsymbol{e}_{i}(=\Delta x / \Delta t)$ is the particle velocity in the $i$ direction and $f_{i}^{e q}$ is the equilibrium single-particle distribution:

$$
f_{i}^{e q}=\rho \omega_{i}\left(1+3\left(\boldsymbol{e}_{i} \cdot \boldsymbol{u}\right)+\frac{9}{2}\left(\boldsymbol{e}_{i} \cdot \boldsymbol{u}\right)^{2}-\frac{3}{2} u^{2}\right),
$$

where $\rho\left(=\Sigma_{i} f_{i}\right)$ is the fluid density, $\boldsymbol{u}\left(\rho \boldsymbol{u}=\Sigma_{i} f_{i} \boldsymbol{e}_{i}\right)$ is the local fluid velocity, and $\omega_{i}$ are the corresponding weights $\left(\omega_{i}\right.$ $=1 / 3$ for $i=0,1 / 18$ for $i=1-6$, and $1 / 36$ for $i=7-18 ; i=0$ corresponds to the rest particle in the center of the cubic lattice, $i=1, \ldots, 6$, correspond to the particles on the axis aligned with $x, y$, and $z$, and $i=7, \ldots, 18$, are related to the particles on the diagonal directions).

The left-hand side of Eq. (1) is the so-called streaming operation, which means that the particles move to the nearest neighbors along their velocity directions (i.e., they radiate from the center of the lattice along their velocity direction). The right-hand side is the collision term, here modeled by the Bhatnagar-Gross-Krook (BGK) collision operator, which describes the redistribution of the particles at each node (for more details see, for example, [10] and [11]). The collisions are entirely local, making the LBM easily and efficiently parallelized.

The choice of the LBM over the classical resolution of the Navier-Stokes equations for the present simulations was motivated by its two important and practical advantages: (i) extreme ease of implementation of complex solid surfaces, and (ii) the local nature of the collision renders the parallelization of any LBM code quite natural and simple. Note that there is no need for solving the Poisson equation for the pressure, which also provides a very significant advantage over the classical direct numerical simulation (DNS).

\section{B. The computational details}

The computational uniform Cartesian grid consists of $800 \times 120 \times 120$ mesh points with $\Delta x=\Delta y=\Delta z$ ( $x$ is the longitudinal direction and $y$ and $z$ are the lateral directions). The

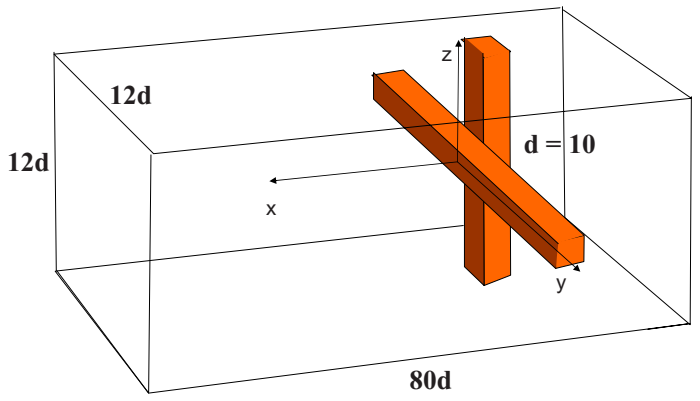

FIG. 1. (Color online) Computational domain with the crossbar.

crossbar (placed at the $x$ node of 100) is made up of two perpendicular square bars in a biplane arrangement; in the present case the second bar is placed perpendicular to the first and rests on its backside (Fig. 1). The width (referred to hereafter as diameter) of the bars, $D$, is represented by ten mesh points. The downstream distance extends to $x / D=70$, where the origin of $x$ is taken at the crossbar location $(y=z$ $=0$ is taken at the centerline).

Periodic conditions were applied in the $y$ and $z$ directions. At the inlet a uniform velocity $\left(U_{0}=0.05\right.$ and $\left.V_{0}=W_{0}=0\right)$ was imposed, and a convective boundary condition was applied at the outlet. To simulate the no-slip condition at the bars, a bounce-back scheme was used; when a particle reaches the wall it bounces back along the same direction at which it arrived.

The Reynolds number, $R_{D}=U_{0} D / \nu$, was about 1600 . This is a relatively small value, which allows a reasonably good computation grid resolution; this latter varied from about $4 \eta$ at $x / D=20$ to $2.5 \eta$ at $x / D=60$ where $\eta$ is the Kolmogorov length scale.

In order to reduce the transient period, a noise was superposed on the initial velocity for a short time. The "steady" state solution was obtained after 10000 iterations. After only the 30000 th iteration were the various statistical quantities calculated over 640000 time steps.

Preliminary calculation revealed that instabilities occurred where the magnitude of the local strain rate,

$$
\Pi_{\alpha \beta}=\frac{1}{2}\left(\frac{\partial u_{\alpha}}{\partial x_{\beta}}+\frac{\partial u_{\beta}}{\partial x_{\alpha}}\right)=\sum_{l} e_{i \alpha} e_{i \beta}\left(f_{i}-f_{i}^{e q}\right),
$$

was large. This was mainly around the crossbar. To help dissipate these instabilities, a large eddy simulation (LES) scheme was introduced:

$$
\nu_{\text {total }}=\nu+\nu_{t},
$$

where $\nu_{t}$ is the turbulent viscosity. The Smagorinsky scheme was used in the simulation. Thus following Hou et al. [12] we have

$$
\nu_{\text {total }}=\nu+(C \Delta)^{2}|S|,
$$

with $C=0.1, \Delta=\Delta x$, and 


$$
|S|=\frac{\sqrt{\nu^{2}+18(C \Delta)^{2}\left(\Pi_{i j} \Pi_{i j}\right)^{1 / 2}}-\nu}{6(C \Delta)^{2}} .
$$

In terms of the time relaxation the LES yields

$$
\tau_{\text {total }}=3\left[\nu^{2}+(C \Delta)^{2}|S|\right]+\frac{1}{2} .
$$

It is important to emphasize that since the LES was used merely as a dissipative scheme for the simulations, no attempt was made to investigate various LES models and their effects on the simulations.

Furthermore, since the LES acted at a subgrid level less than or equal to $\Delta x$, it was believed that the LES model did not change significantly the results concerning motions whose scales were larger than a few times the Kolmogorov length scale (see discussion in the next section below).

The calculations were carried out on a cluster of 16 dual processors (effectively 32 processors each at $2.4 \mathrm{GHz}$ ). The MPI2 protocol was used to perform the parallelization.

\section{RESULTS}

\section{A. Comparison between LBM simulations and PIV and LDV measurements}

The computational mesh resolution $(2 \eta<\Delta x<4 \eta)$ was of similar quality than many other DNSs based on the Navier-Stokes equations (NS-DNS). In that regard, the LBM simulation can also be considered as a DNS based on the lattice Boltzmann method (LBM-DNS). However, because a LES Smagorinsky scheme with a filter $\Delta=\Delta x$ was used to control the numerical instabilities (mainly around the crossbar), one had to be careful, particularly in the near-wall region where it is known that the Smagorinsky scheme is not accurate. Thus in order to determine the adequacy and validity of the LBM-DNS we compared the numerical results with experimental data.

Particle image velocimetry (PIV) measurements were carried out in a free surface water tunnel. The test section was 8 $\mathrm{m}$ long, $60 \mathrm{~cm}$ wide, and $60 \mathrm{~cm}$ deep. The water depth $(h$ $=50 \mathrm{~cm})$ and the flow rate $\left(94 \mathrm{~m}^{3} / \mathrm{h}\right)$ were kept constant. The free surface was relatively calm with no waves occurring at the surface. A biplane crossbar made of two square bars was mounted at about $3 \mathrm{~m}$ downstream of the inlet of the working section. The "diameter" of the bars was $2 \mathrm{~cm}$ which gave a Reynolds number, $R_{D}$, of about 1600 . A $200 \mathrm{~mJ}$ pulsed Nd:Yag was used to illuminate the flow. The laser sheet was shot from the top through a $10 \mathrm{~cm}$ diameter Perspex porthole. The porthole was flush with the free surface in order not to disturb the flow. A set of cylindrical lenses converted the laser light into a vertical thin sheet located at midplane $(z=0)$. A digital camera (Kodak ES 1.0) was used with a charge-coupled device (CCD) (1008 pixels $\times 1008$ pixels). The PIV images were post-processed using the adaptive-correlation method (FlowManager 4.30.27; Dantec Dynamics) to obtain the instantaneous velocity fields. Each image, which corresponded to an area of about $6 \mathrm{~cm}$ $\times 6 \mathrm{~cm}$ of the actual flow, was subdivided into 32 pixels $\times 32$ pixels with $50 \%$ overlap. The water was seeded with particles (Optimage Ltd.) with an average size of $30 \mu \mathrm{m}$ and a specific gravity of $1.0 \pm 0.02$. These particles were polycrystalline in structure and provided a high light-scattering efficiency (five times greater than latex spheres which have a similar refractive index).

Figure 2 shows examples of computed (left) and measured (right) velocity fields at $z=3 D$ (top) and $z=0.1 D$ (bottom). In the figure, $y$ is in the vertical direction and $x$ and $z$ are in the horizontal plane and the flow is from left to right. In the top figures, only a region around the upper part of a cylinder is shown to better visualize the velocity field. The PIV velocity field shown in the bottom right figure is taken at a $z$ plane located very near the surface of the vertical bar (delimited by the vertical solid lines); it was difficult to measure the exact position of the laser light sheet.

Clearly the LBM-DNS velocity fields present similar features to those observed in the PIV data. At $z=3 D$ the PIV data show a counterclockwise vortex (illustrated by a streamline) taking place over the bar. It was observed in the PIV film sequence that these vortices occurred much less frequently than the clockwise vortices. It is for this reason that this velocity field is selected for comparison with the simulation. The figure on the left clearly shows that the LBMDNS reproduces these rare counterclockwise vortices with reasonable accuracy. The simulation reproduces also the upward vertical motion and the large clockwise vortical structure visible in the PIV data. While not shown here, it was verified that the simulation faithfully reproduced the flow features around a bar as observed in the PIV data. For example, the clockwise vortices generated at the wall and their shedding were well reproduced in the LBM-DNS simulation. At $z=0.1 D$, the lateral motions observed close to the vertical bar in the PIV velocity field are also observed in the LBMDNS data. The flow behind the vertical bar where vortical structures are visible in the LBM-DNS is very much similar to that observed in the PIV data. It is further encouraging and satisfying to observe that the scales of the computed vortical structures are of similar length than those seen in the PIV data.

The LBM-DNS simulations were further assessed with laser Doppler velocity (LDV) measurements. The LDV have been carried out in a constant-head closed circuit vertical water tunnel with a $2 \mathrm{~cm}$ long square $(250 \mathrm{~mm}$ $\times 250 \mathrm{~mm}$ ) Perspex (20 mm thick). The tunnel was free of any problem arising from contamination, pumps, and vibrations. A biplane crossbar made of two (Perspex) square bars was mounted at about $1.3 \mathrm{~m}$ downstream of the inlet of the working section. The diameter of the bars was $11 \mathrm{~mm}$ yielding a Reynolds number $R_{D}$ of about 1600 when the velocity upstream of the crossbar was about $0.15 \mathrm{~m} / \mathrm{s}$. The freestream turbulence intensity at that velocity was about $3 \%$. The LDV measurements were carried out with a threecomponent fiber-optic LDV system (Dantec). The laser source was a $5 \mathrm{~W}$ Ar ion. A two color probe was used in forward scatter mode to measure simultaneously the streamwise and transverse components of the velocity. The measuring volumes were about $0.063 \mathrm{~mm} \times 0.063 \mathrm{~mm}$ $\times 0.47 \mathrm{~mm}$ each. The data acquisition was made with two Dantec enhanced burst spectrum analyzers (BSAs) to which the photomultiplier signals entered. The data rate was typi- 

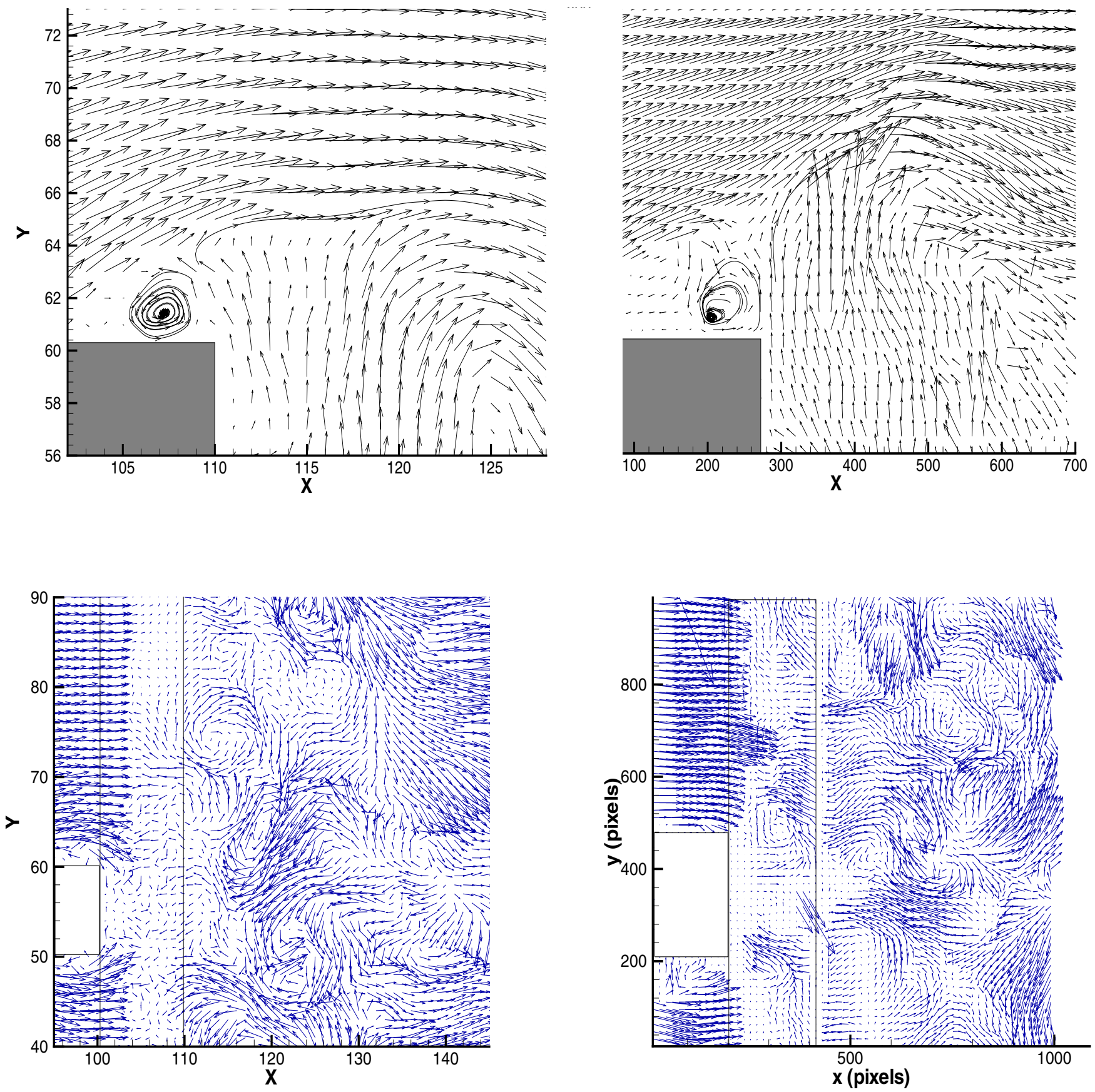

FIG. 2. (Color online) Computed (left) and measured (right) instantaneous velocity fields. Left: $z=3 D$, bottom: $z=0.1 D$.

cally about $450 \mathrm{~Hz}$. About 30000 samples were collected at each data point. No artificial seeding was used as the natural seeding of the water was found sufficient for the measurements. The effect of the velocity bias was corrected by weighing individual realizations with the transit time of particles in the measuring volumes [13].

Figure 3 presents both the LBM-DNS and LDV data for the mean velocity $(U)$ and the streamwise $\left(u^{\prime}\right)$ and transverse $\left(w^{\prime}\right)$ fluctuating components of the velocity along the centerline of the crossbar wake (the prime denotes the rms). The data are normalized by the mean velocity $U_{0}$ taken upstream of the crossbar. There is a general good agreement between the LBM-DNS and LDV results. For example, the stream- wise location of minimum of $U$ and the magnitude of the minimum are well reproduced in the LBM-DNS data. Of particular interest is also the very good reproduction of the streamwise locations of the maximum in $u^{\prime}$ and $w^{\prime}$ (a log-log scale was used). Furthermore and quite remarkably, the calculated $w^{\prime}$ profile follows closely the measured one. There are, however, some discrepancies between the LBM-DNS and LDV data. The recovery of $U$ after its minimum is slightly underpredicted by the LBM-DNS and the measured $u^{\prime}$ profile appears to be systematically higher than the calculated one. Also, while the LBM-DNS captures well the $x$ location of the first crossover between $u^{\prime}$ and $w^{\prime}$, it predicts the second crossover at $x / D$ larger than that found in the 

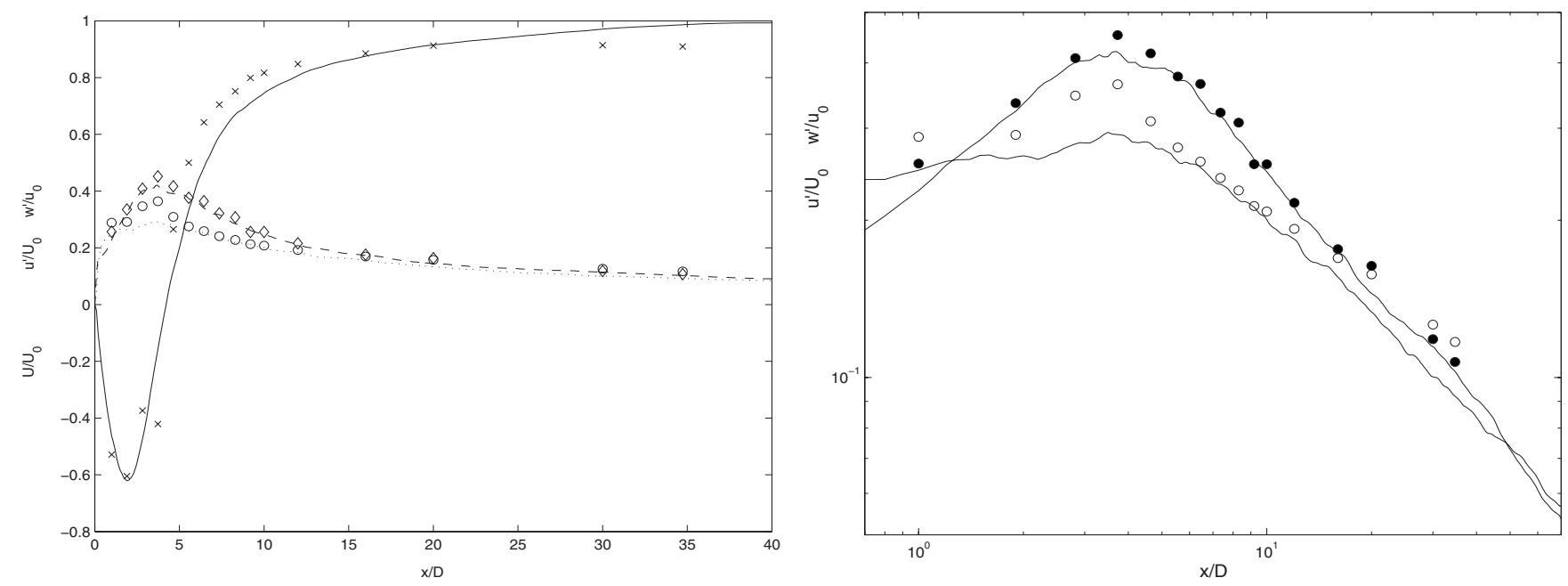

FIG. 3. Computed (lines) and measured (symbols) mean velocity and velocity fluctuation rms along the centerline. Left figure: lines, solid: $U / U_{0}$, dashed $w^{\prime} / U_{0}$, dotted: $u^{\prime} / U_{0}$; symbols, crosses: $U / U_{0}$, diamonds: $w^{\prime} / U_{0}$, circles: $u^{\prime} / U_{0}$. Right figure: solid circles: $w^{\prime} / U_{0}$, open circles: $u^{\prime} / U_{0}$.

LDV data. No clear reasons can be put forward at this stage as to why the predicted $u^{\prime}$ presents some variations from the measurements. On the other hand, the change in the slope in the decay of $u^{\prime}$ and $w^{\prime}$ observed in the LDV data at $x / D$ of about 15 is also discernable in the LBM-DNS data, particularly in $w^{\prime}$, although at a later $x / D$.

To summarize the comparison between the LBM-DNS and PIV and LDV data, one can argue that the LBM-DNS predicts properly the near-wall flow with sufficiently resolved details. In particular, the vortical structures as well as the transverse motions over the surface of the crossbar are well reproduced. This provides confidence in the simulation and indicates that the LES used for stability control only does not introduce erroneous results at scales equivalent to the mesh resolution, even in the region near the bars. Furthermore, the good agreement between the LBM-DNS results and PIV data shows that the mesh resolution is fine enough for the simulation to capture well the details of the vortical structures that are relevant for the subsequent development of the wake behind the crossbar. This, in turn, ensures that the simulated wakes and their interactions are a faithful representation of what can be observed experimentally.

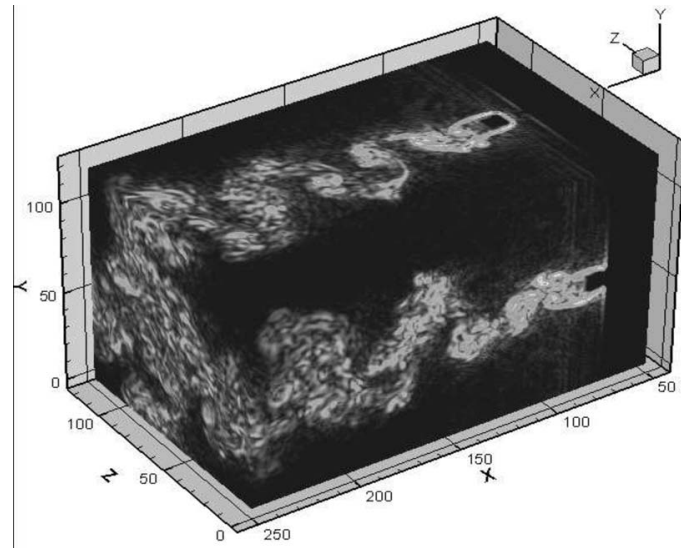

\section{B. Numerical flow visualization}

Figure 4 shows an example of the simulated flow. It represents isocontours of the instantaneous enstrophy, $\omega^{2}(\omega$ is the vorticity magnitude), in the region $x / D<15$ behind the crossbar. A cut was made in the bottom figure to reveal the half spanwise extent of the wake of each bar. Notice the biplane arrangement of the square bars, which causes a shift in the streamwise direction between the two wakes.

Away from the centerline the wakes exhibit the characteristics of two-dimensional wakes behind square cylinders. The vortex shedding, for example, is clearly visible in the top view. However, while away from the centerline the twodimensional wakes tend to keep their identity, they interact strongly in the central region, losing their individual characteristics. This is well illustrated in Fig. 5 which shows the spectra of the $w$ component of the velocity at $y / D=1$ and 3 for $z / D=1$ and $x / D=5$. For the outermost position $(y / D$ $=3$ ), one can observe a peak in the spectrum corresponding to the shedding frequency of the cylinder with a Strouhal number St $\left(=f D / U_{0}, f\right.$ is the shedding frequency) of about 0.16 . Quite interestingly, this value is close to that $(\mathrm{St}$ $=0.17$ ) obtained in a grid-generated turbulence where the

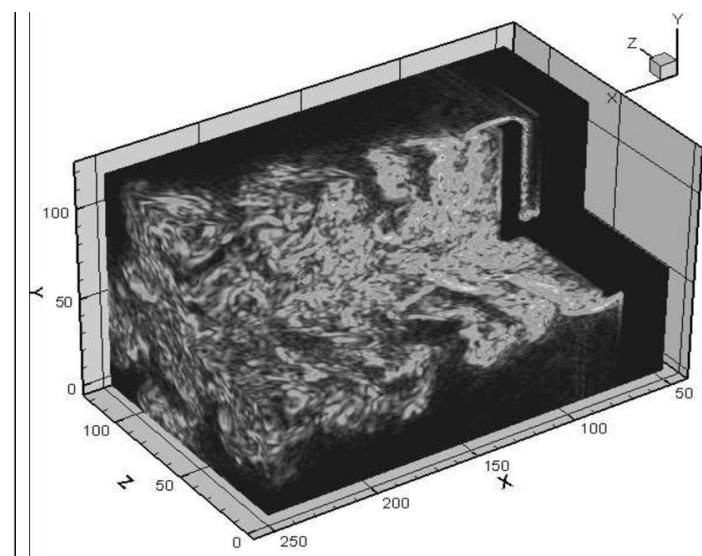

FIG. 4. Contours of $\omega^{2}$ right view shows a cutout $(0 \leq y \leq 60,0 \leq z \leq 60)$ to reveal the interaction of the individual wakes. 


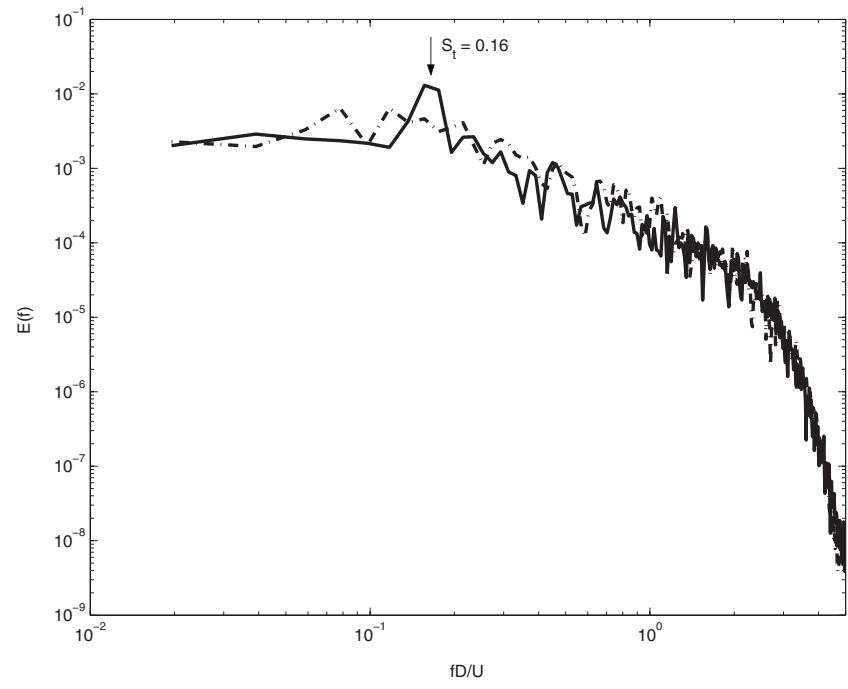

FIG. 5. Spanwise velocity spectra at $z / D=1, x / D=5$. Dotdashed line: $y / D=1$; solid line: $y / D=3$.

grid is made of biplane square bars [4]. This supports the idea that the flow behind the crossbar may present similar features to that of a grid-generated turbulence just behind the grid and before the wakes of successive bars interact, thus reinforcing the argument that the crossbar arrangement may be considered as the unit element of grid made of bars. An interesting study would be to determine the shedding modes (in phase or out of phase) between the individual wakes and whether an interlocking mode would take place, and how the subsequent turbulence decay would be altered by the shedding mode.

As illustrated in Fig. 4, the near-wake of the crossbar is populated by relatively strong coherent vortical structures. This was already seen in Fig. 2 which reveals vortices whose axis are aligned with $y$ or $z$ directions. What is remarkable in Fig. 2 is the presence of lateral motions near the bar. To study these further, Fig. 6 shows the flow in the $(x, y)$ plane at $z / D=-0.7$ and $z / D=-1.2$ (i.e., near both sides of the vertical bar); the region shown starts at the leading edge of the vertical bar and ends at a distance of about five times the bar diameter. There is a pseudoperiodic flow pattern in the form of streaks along the bar. While the streaks mark regions of

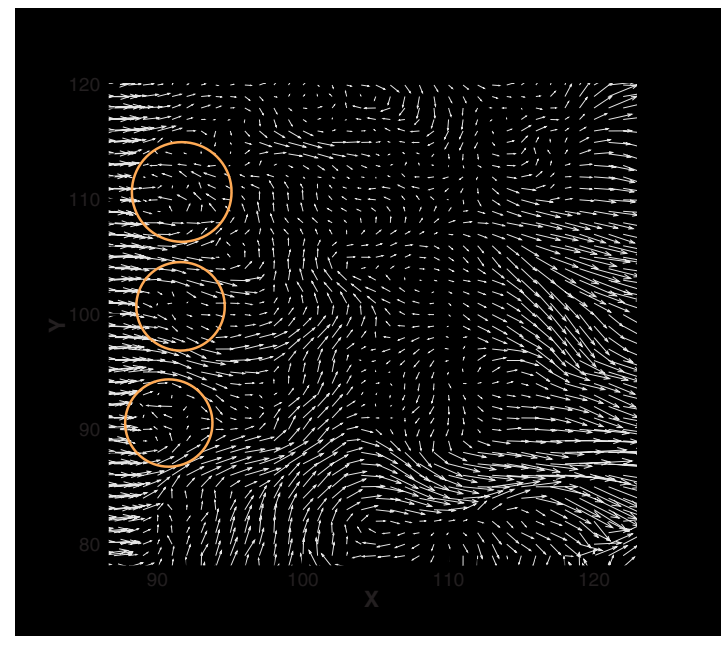

FIG. 7. (Color online) Closer view of top image of Fig. 6. The circles highlight examples of regions of spanwise motions along the bar.

high speeds, they are somehow reminiscent of the low-speed streaks observed in the near-wall region of a smooth wall turbulent flow. Although no attempt has been made to determine the average wavelength between the streaks, one may infer from the figure that the spacing is likely to be between $0.5 D$ and $D$.

Examination of the right image in Fig. 6 suggests that a "streak shedding" is taking place. Indeed, on that image streaks are clearly visible at the bar and at $x / D$ of about 2 , while in the left image they are clearly defined from the bar up to $x / D=1$. Thus arguing that the streaks are associated to coherent structures-different from the "classical" vortices generated at the cylinder which form the Von-Karman streets-Fig. 6 would indicate that the vortex shedding is accompanied by shedding of many vortical structures originating at the crossbar.

While the mechanism for the formation of these streaks is not clear, a closer look around these streaks (Fig. 7) reveals that they result from the occurrence of localized spanwise motions along the bar. These motions reflect intermittent recirculatory motions as clearly evident in Fig. 8 which presents the instantaneous isocontours of the $z$ component of the
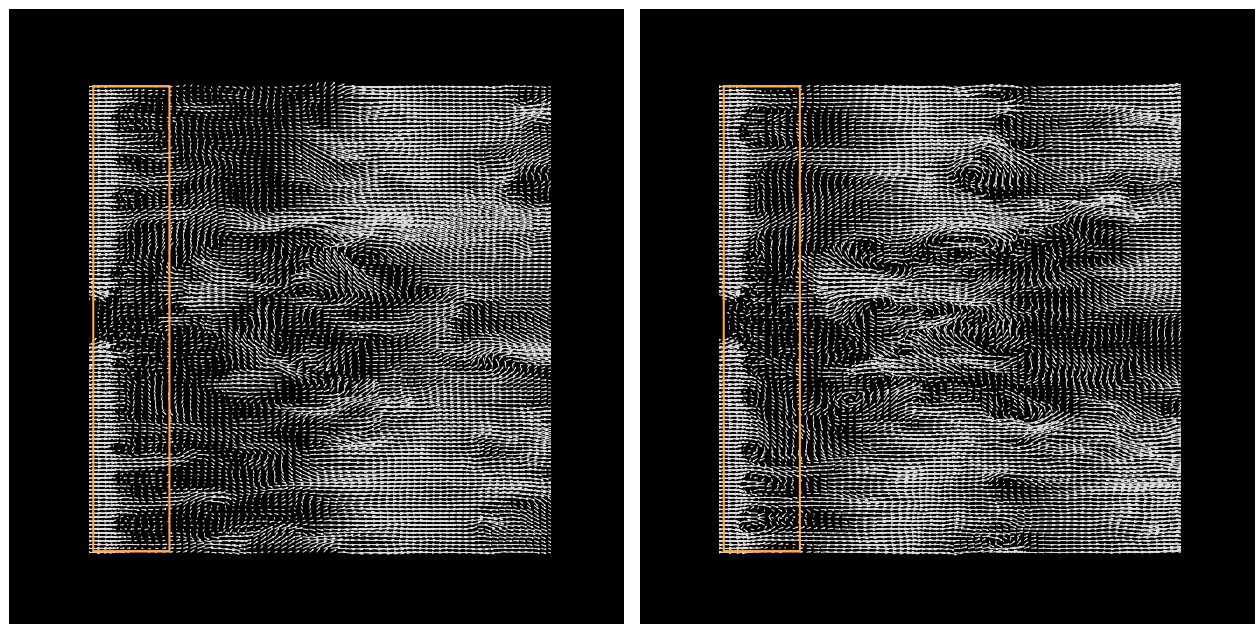

FIG. 6. (Color online) Instantaneous velocity field in the $(x, y)$ plane. Left image: below the bar, $z / D=-0.7$; right image: above the bar, $z / D=1.2$. The vertical bar's silhouette is represented by the white closed line. 


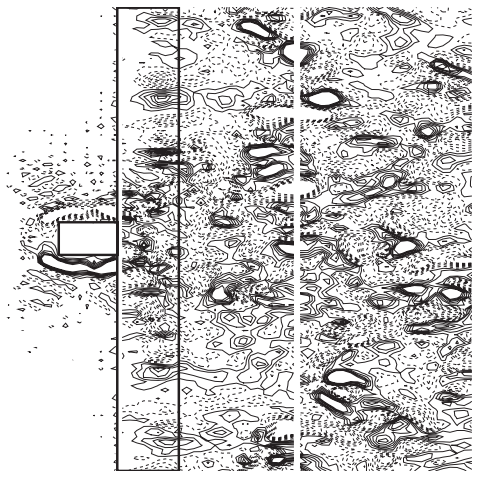

vorticity, $\omega_{z}$. In the figure, the ratio $\omega_{z} / \omega_{z, \max }$, where $\omega_{z, \max }$ is the maximum of $\omega_{z}$, is shown. One can clearly see positive and negative $\omega_{z}$ along the bar. Furthermore, the occurrence of $\omega_{z}$ forms a pattern consisting of pairs of positive and negative vorticity. Notice that an average spacing between the pairs appears to be about $0.5 D$ and $D$. The figure suggests that these localized and relatively intense $\omega_{z}$ are shed (certainly during the vortex shedding process), thus feeding the earlier stages of the wake of the crossbar with $v^{\prime}$ [14]. Considering that the flow around the second bar should have features similar to those shown in Fig. 8, one can expect $w^{\prime}$ to be also injected into the wake.

The development of the flow behind the crossbar is observed in Fig. 9, which shows the instantaneous velocity field
FIG. 8. Instantaneous isocontours of $\omega_{z} / \omega_{z \text {, max }}$ on both sides of the vertical bar; the range of $\omega_{z} / \omega_{z, \max }$ shown is $[-0.3,0.3]$. Dashed lines correspond to negative $\omega_{z}$
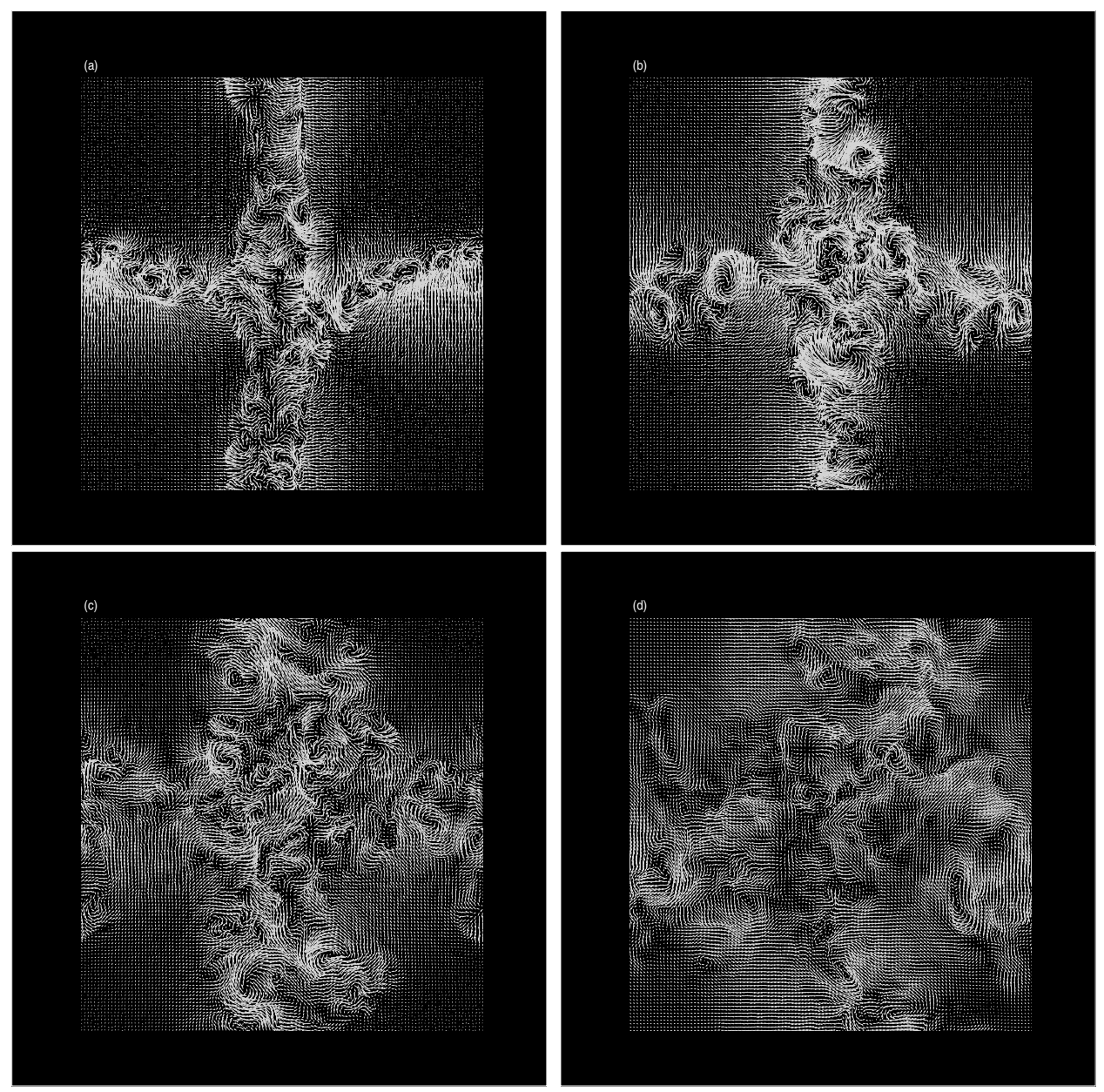

in the $(z, y)$ plane at four positions downstream of the crossbar $(x / D=1,3,7$, and 14). Close to the crossbar [Figs. 9(a) and $9(\mathrm{~b})$ ], one can see vortical motions developing along the span of the two bars. Such motions, which form a pattern of positive and negative streamwise vorticity, have been observed and documented in the literature-in particular Figs. 2-4 of Lin et al. [15] show similar vortices behind a circular cylinder. These motions reflect concentrations of streamwise vorticity $\left(\omega_{x}\right)$ along the bars and mark the presence of streamwise vortical structures. Note that, as they increase in size with the downstream distance, they inevitably interact leading to their destruction, or at least reduction in their coherence. The figure reveals the effect of the interaction of the two individual bars' wakes on the velocity field as the dis-
FIG. 9. Instantaneous velocity field in the $(z, y)$ plane; (a) $x / D$ $=1$, (b) $x / D=3$, (c) $x / D=7$, and (d) $x / D=14$. 

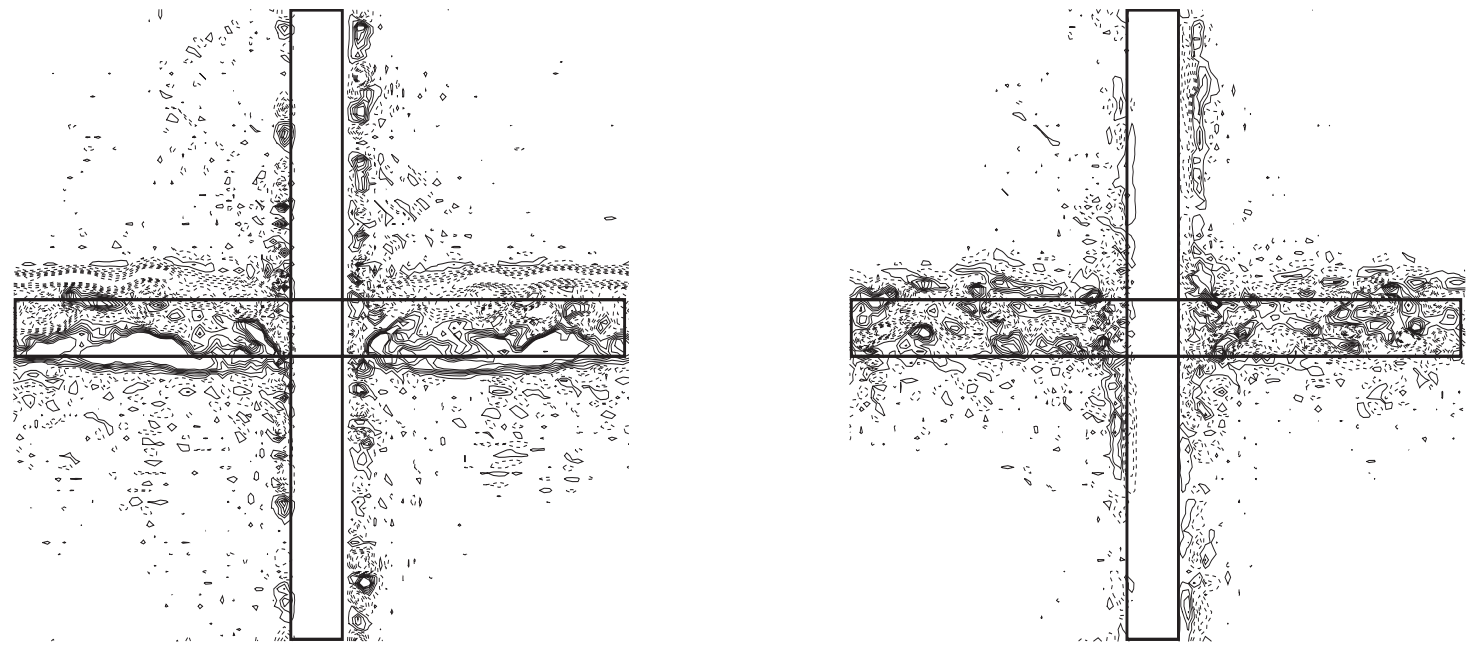

FIG. 10. Instantaneous isocontours of $\omega_{z} / \omega_{z, \max }$ (left figure), and $\omega_{x} / \omega_{x, \max }$ (right figure) in the $(y, z)$ plane at $x / D=-0.5$; Dashed lines correspond to negative values.

tance $x / D$ increases. The two wakes, well identified for $x / D \leq 3$, becomes less identifiable at $x / D=7$; at $x / D=14$ they have merged to form a single wake, which occupies almost the entire computational domain across the whole $(z, y)$ plan (only the four corners show little activities); for $x / D \geq 20$ (not shown here) the wake occupies fully the plane. The sequence of velocity fields shows that the crossbar wake will eventually become axisymmetric and would present features similar to that of a grid-generated turbulence. In particular, the velocity fields for $x / D \geq 20$ are similar to those found in DNS results of a grid-generated turbulence [11].

It is beyond the scope of the present paper to discuss further the characteristics of these streamwise structures (the reader may consult [15] for such discussion). However, one may provide a tentative explanation for their formation. Let us consider the vertical bar. As seen in Fig. 8, the bar is a source of a relatively strong intermittent $v$ component through the intermittent spanwise recirculatory motions taking place at the bar. The bar also generates a $w$ component, associated with the vorticity component aligned with the bar

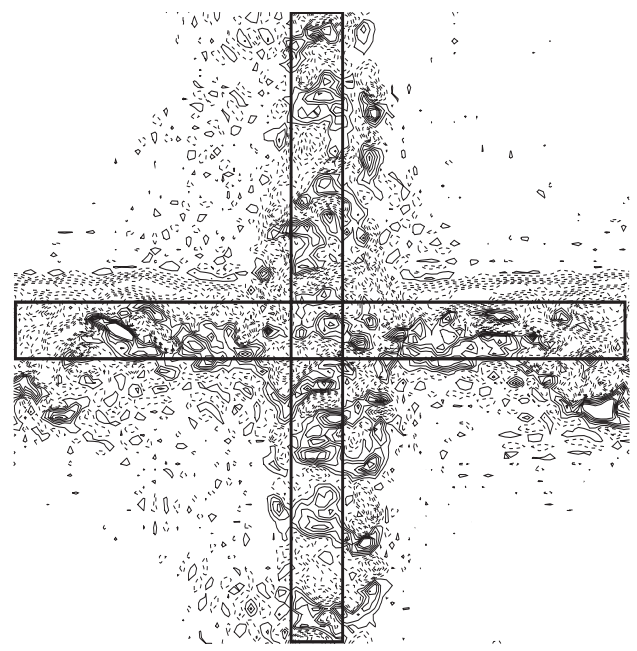

axis, in this case $\omega_{y}$. One can argue that such pattern leads to the formation of streamwise vorticity $\omega_{x}$. This scenario would be similar to that occurring during the process leading to the 3D transition to turbulence in a cylinder wake at low Reynolds numbers [16], which yields to the $A$ mode [17]. Accordingly, it appears that $\omega_{z}$ is a crucial ingredient for the generation of $\omega_{x}$. This is seen in Figs. 10 and 11 showing instantaneous isocontours of both $\omega_{z}$ and $\omega_{x}$ in the $(z, y)$ plane at two streamwise locations, $x / D=-0.5$ and 0.5 , respectively (the plane at $x / D=-0.5$ is at the middle of the vertical bar). In the figure, the flow is pointing out of the page and the vertical bar is downstream of the horizontal bar (i.e., closer to the reader). There is a relatively strong correlation between the locations of $\omega_{z}$ and those of $\omega_{x}$ along the vertical bar. This is particularly more significant in Fig. 11 than in Fig. 10. For example, along the vertical bar, where there is an almost homogeneous input of $w$ component due to $\omega_{y}$, the occurrence of $v$ related to the intermittent presence of spanwise vorticity yields the creation of $\omega_{x}$. Of course, a similar mechanism is at play along the horizontal bar result-

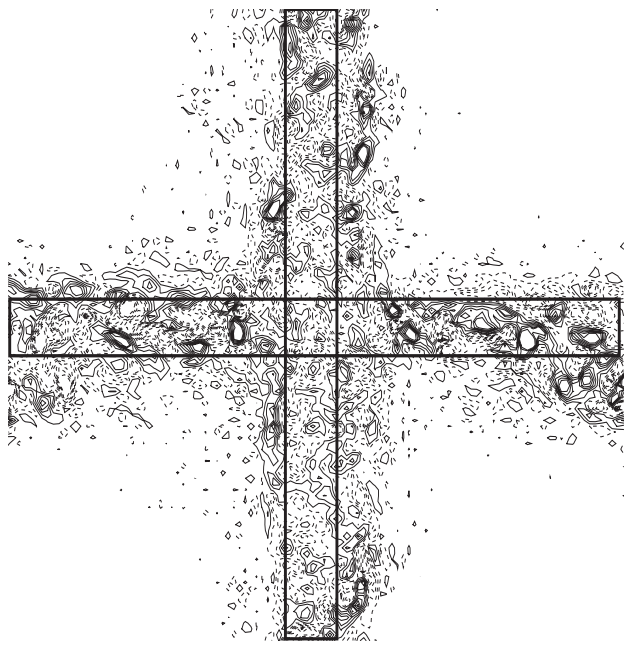

FIG. 11. Instantaneous isocontours of $\omega_{z} / \omega_{z, \max }$ (left figure), and $\omega_{x} / \omega_{x, \max }$ (right figure) in the $(y, z)$ plane at $x / D=0.5$; Dashed lines correspond to negative values. 

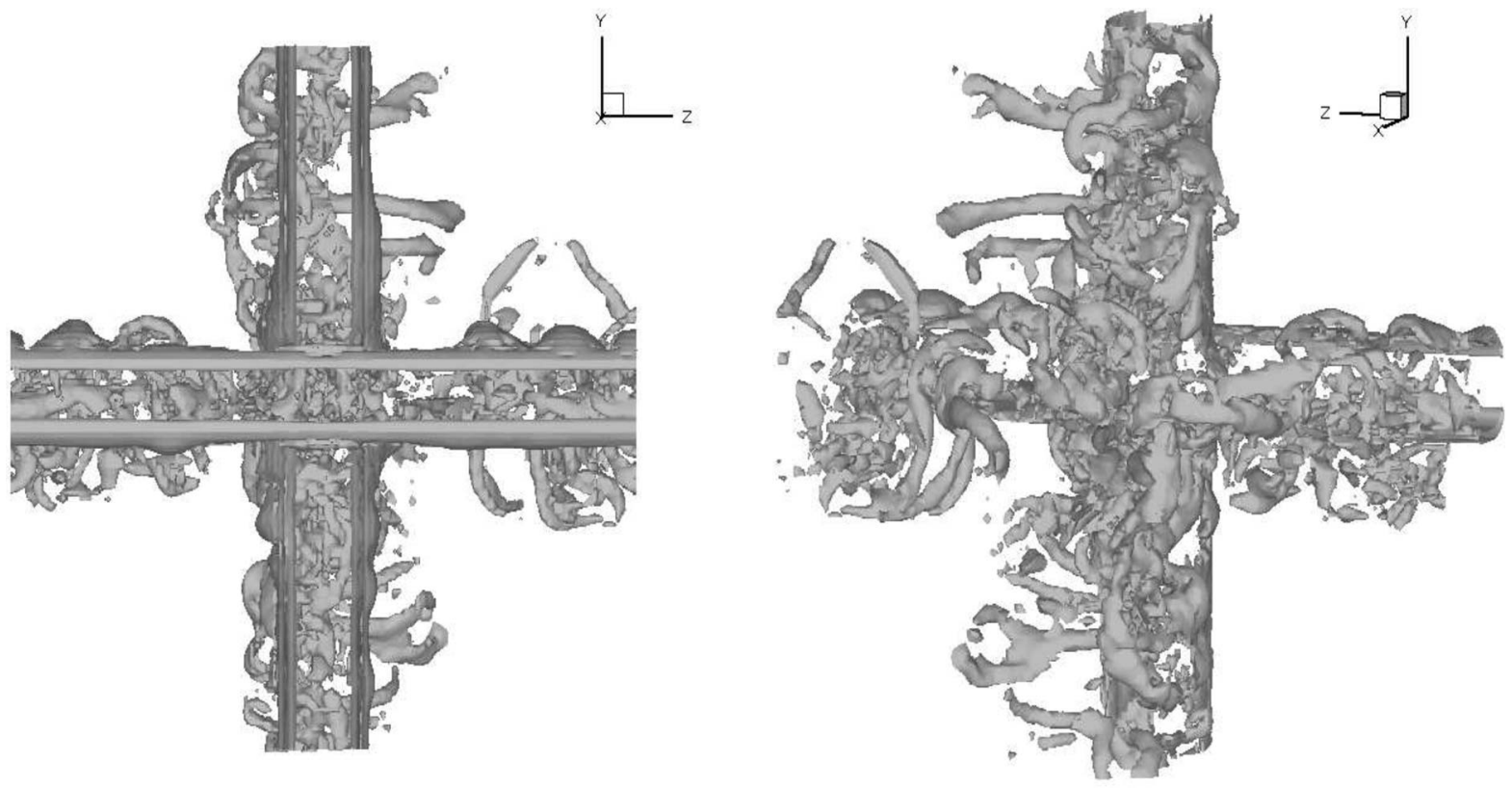

FIG. 12. Instantaneous contour of $|\omega| /\left|\omega_{\max }\right|=0.3$. Left: front view, right: back view.

ing in generation of $\omega_{x}$ along that bar. There, $\omega_{y}$ plays the role that $\omega_{z}$ does for the vertical bar.

A vivid description of the structures behind the crossbar is given in Fig. 12 showing a three-dimensional representation of an isocontour of vorticity (magnitude ratio $|\omega| /\left|\omega_{\max }\right|$ $=0.3$ ) in the region $-2.5 \leq x / D \leq 4$. Two view angles are presented: front or downstream view (the flow is into the paper; left image) and backside or upstream side view (the flow is out of the paper and to the left; right image). In the front view, we can clearly distinguish blobs of vorticity along the sides of the bars - these are most clearly defined on the topside of the horizontal bar and also visible in the backside view. These blobs are "resting" on top of the vorticity sheet attached at the surface of the bars. Behind the crossbar the flow is dominated by elongated and deformed interlaced structures. The interlacing reflects the strong nonlinear interactions between the various coherent vortical structures and their stretching which leads to the production of turbulence in this region of the near-wake (as can be observed in Fig. 3).

\section{Decay of turbulence}

The previous section showed that the region of the nearwake comprised between $0 \leq x / D \leq 4-5$ is populated by streamwise and transverse vortical structures. It is important to note that the individual wakes are still clearly defined in this region, suggesting that they do not interact (apart from the central region of the crossbar). However, as the distance $x / D$ increases the two wakes begin to interact leading to one common wake, which bears a similarity to grid-generated turbulence wake (see Fig. 9). It is then interesting to look at the decay of turbulence in this wake.

Figure 13 shows the variations along the centerline of the normal Reynolds stresses, $\left\langle u^{2}\right\rangle,\left\langle v^{2}\right\rangle$, and $\left\langle w^{2}\right\rangle$, normalized by the upstream velocity $U_{0}(u, v$, and $w$ are the $x, y$, and $z$ components of the velocity, respectively). Behind the cross- bar, turbulence is first generated as reflected in the increase of all three Reynolds stresses. It peaks to a maximum when $x / D$ is about $3-4$, then decreases. Throughout this variation the distribution of the turbulent kinetic energy among its components changes. Just behind the crossbar, $\left\langle u^{2}\right\rangle$ is larger than $\left\langle v^{2}\right\rangle$ which in turn is larger than $\left\langle w^{2}\right\rangle$ until about $x / D$ $=0.6$. Then $\left\langle w^{2}\right\rangle$ becomes the largest and $\left\langle u^{2}\right\rangle$ the smallest. Notice the relatively sharp increase in $\left\langle v^{2}\right\rangle$ and $\left\langle w^{2}\right\rangle$, with a peak occurring at about $x / D=3$. The $x / D$ region of high values of $\left\langle v^{2}\right\rangle$ and $\left\langle w^{2}\right\rangle$ is comprised between 1 and 6 . It is interesting to point out that the $\left\langle u^{2}\right\rangle$ data compare favorably with those taken in a near-field of a grid-generated turbulence [18].

There are interesting features exhibited by the data in Fig. 13: (i) an inflexion point is seen at $x / D \approx 30$ in the $\left\langle w^{2}\right\rangle$

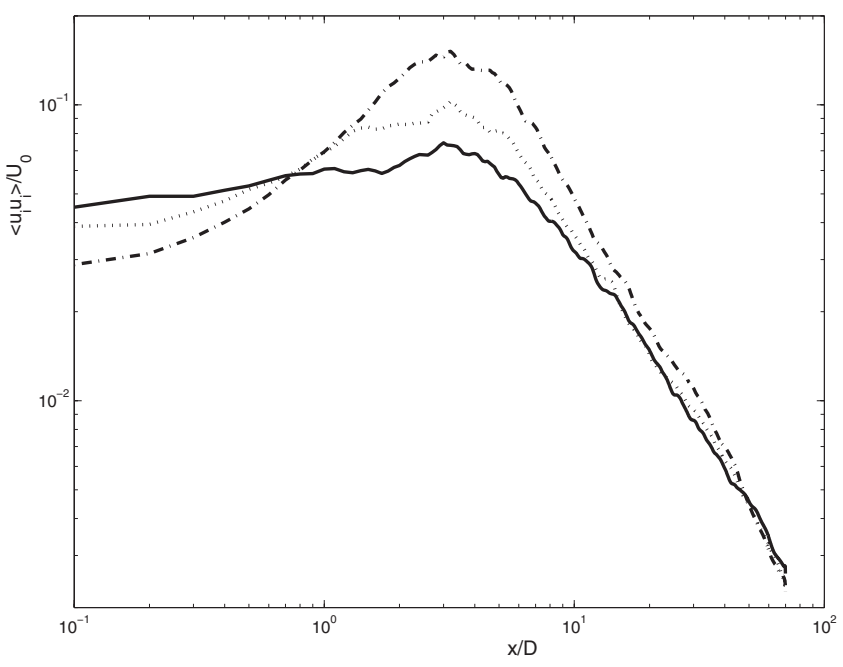

FIG. 13. Streamwise variation of the normal Reynolds stresses along the crossbar centerline. Solid line: $\left\langle u^{2}\right\rangle$, dotted line: $\left\langle v^{2}\right\rangle$, dotdashed line: $\left\langle w^{2}\right\rangle$. 


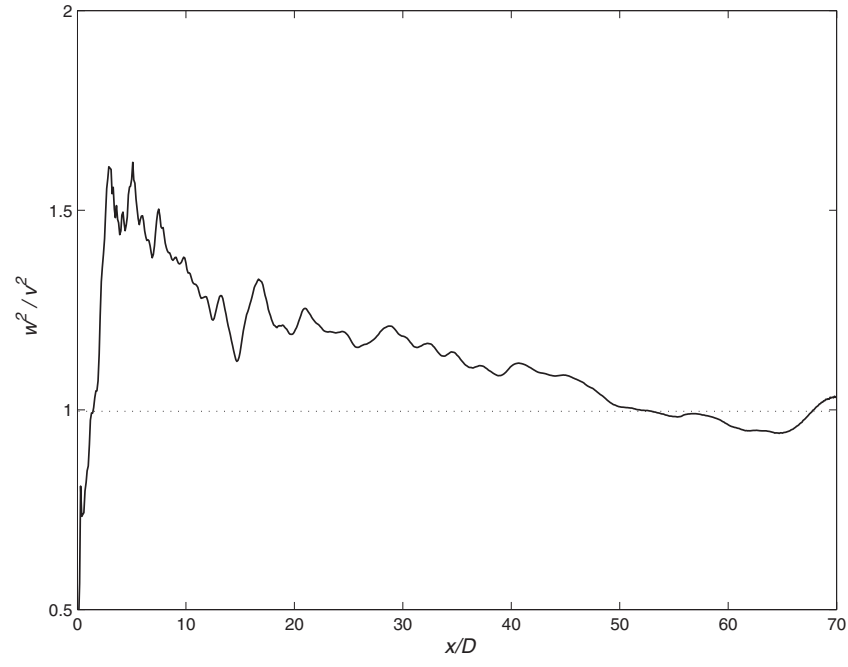

FIG. 14. Streamwise variation of the ratio $\left\langle w^{2}\right\rangle /\left\langle v^{2}\right\rangle$ along the crossbar centerline.

curve; while not clearly visible with the figure scale, a similar inflexion point is also observed in the $\left\langle v^{2}\right\rangle$ distribution; (ii) there is a crossover at $x / D \approx 45$ beyond which $\left\langle u^{2}\right\rangle$ becomes the largest component. Point (i) suggests a transition in the turbulence state resulting in a change in the redistribution of the turbulent kinetic energy. The change is such that more energy is transferred to $\left\langle u^{2}\right\rangle$ which will lead to feature (ii) and $\left\langle u^{2}\right\rangle$ remaining the largest component after the crossover and during the subsequent decay. The ratio $\left\langle u^{2}\right\rangle /\left\langle w^{2}\right\rangle$ is about 1.10 for $x / D>50$. However, there is not enough downstream development to determine whether this ratio remains constant or increases.

The fact that $\left\langle w^{2}\right\rangle$ is larger than $\left\langle v^{2}\right\rangle$ is related to the present arrangement of the two cylinders: the second cylinder, perpendicular to the direction $z$, promotes more $\left\langle w^{2}\right\rangle$ than $\left\langle v^{2}\right\rangle$. While $\omega_{y}$ and $\omega_{z}$ dominate along their respective axes, $\omega_{y}$ shows a stronger intensity. Furthermore, $\omega_{y}$ predominates around the central region $\left(\omega_{z}\right.$ is comparatively nonexistent there). Since the velocity fluctuations in the $\mathrm{z}$ direction are related to the vorticity in the direction perpendicular to that axis [14], in this case $\omega_{y}$, one can then expect to observe a difference in the lateral velocity fluctuations close to the crossbar. Had the two bars been in the same plane no such bias would be occurring; $\left\langle w^{2}\right\rangle$ and $\left\langle v^{2}\right\rangle$ would have received the same amount of energy to the detriment of $\left\langle u^{2}\right\rangle$.

Figure 13 reveals also an important result, namely that a biplane arrangement is a source of anisotropy. This is emphasized in Fig. 14 showing the ratio $\left\langle w^{2}\right\rangle /\left\langle v^{2}\right\rangle$ along the centerline of the crossbar. $\left\langle w^{2}\right\rangle$ is larger than $\left\langle v^{2}\right\rangle$ over a long distance; only when $x / D>45$ (at the crossover point) do the two velocity fluctuation components become approximately equal. For a square-bar biplane grid, with a mesh size of $M=5 D$, the ratio $\left\langle w^{2}\right\rangle /\left\langle v^{2}\right\rangle$ is found to approximate 1 when $x / M>15$, that is, $x / D>75[5]$.

The streamwise variations of the probability density function (pdf) for each velocity component were calculated (not shown here). All the pdf's followed the Gaussian distribution

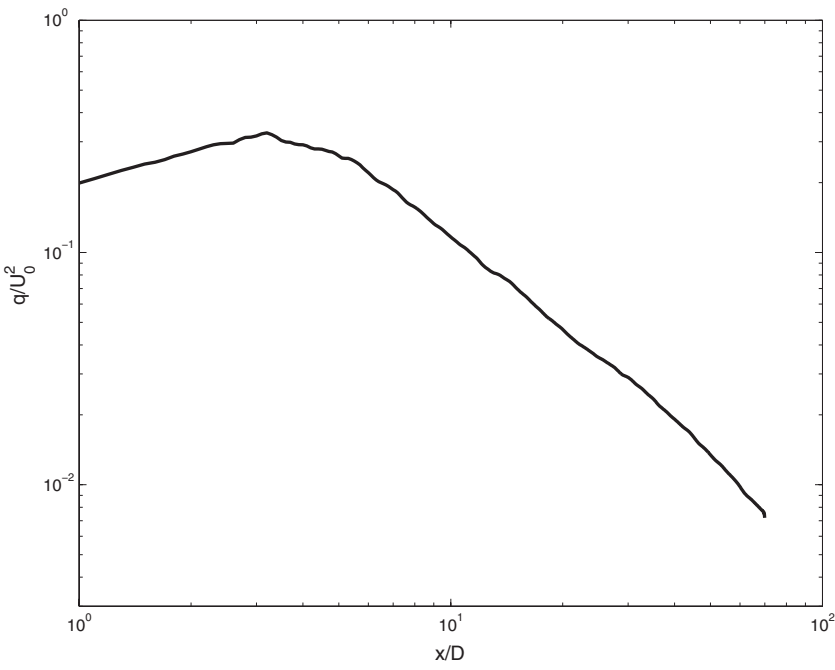

FIG. 15. Streamwise variation of the turbulent kinetic energy along the crossbar centerline.

with some variations, as reflected in the values of the velocity skewness $S_{u}=\left\langle u^{3}\right\rangle /\left\langle u^{2}\right\rangle^{3 / 2}$ and kurtosis $K_{u}=\left\langle u^{4}\right\rangle /\left\langle u^{2}\right\rangle^{2}$ $\left[\left(S_{u}, K_{u}\right)=(0.19,3.06),(0.096,3.15),(0.262,3.12) ; \quad\left(S_{v}, K_{v}\right)\right.$ $=(-0.087,3.12),(0.026,3.24),(-0.15,3.24) ; \quad\left(S_{w}, K_{w}\right)$ $=(0.0052,2.6),(-0.0375,2.96),(-0.057,2.96)$ for $x / D=5$, 20 , and 60 , respectively]. The $u$ pdf was slightly skewed toward the positive values, while the pdf of $v$ was slightly skewed toward the negative values. The $w$ pdf appeared more symmetric than the $u$ and $v$ pdf's. Note that had the two bars be in an in-plane arrangement the $v$ and $w$ pdf's would have been similar. The observed variations reflected the biplane arrangement. Of course the pdf's should eventually evolve toward a Gaussian distribution as $x / D$ increases. The results merely indicated that the evolution of the pdf's in the range $0<x / D<60$ illustrated the interaction between the bar wakes. Jayesh and Warhaft [18] showed that at $x / M=1$ behind a grid made of square bars the pdf of $u$ is strongly dependant of the probe lateral position. However, by $x / M$ $=4$, the pdf becomes almost Gaussian irrespective of the probe position. In the light of the numerical flow visualization results presented in Sec. III B, one expects that the pdf's in the $x / D$ region close to the crossbar to be strongly dependant of the $y$ and $z$ positions. Future LBM-DNS will be carried out to investigate this assessment.

The decay of the turbulence kinetic energy is observed in Fig. 15, which shows the variation of $q / U_{0}^{2}$ along the centerline of the crossbar. The turbulence starts decaying at $x / D$ about 3-4. The decay of $q / U_{0}^{2}$ reflects that of its components. In particular, the inflexion point, observed in $\left\langle w^{2}\right\rangle$, is clearly visible at $x / D \approx 30$, indicating that $\left\langle w^{2}\right\rangle$ contributes more to the turbulent kinetic energy than $\left\langle u^{2}\right\rangle$, thus imposing its decay in the early stage of the turbulence decay.

Figure 16 shows the decay of the turbulent kinetic energy dissipation rate,

$$
\epsilon_{\text {true }}=\overline{\nu \frac{\partial u_{i}}{\partial x_{j}}\left(\frac{\partial u_{i}}{\partial x_{j}}+\frac{\partial u_{j}}{\partial x_{i}}\right)},
$$

along the centerline of the crossbar. The decay of the locally isotropic $\epsilon$, 


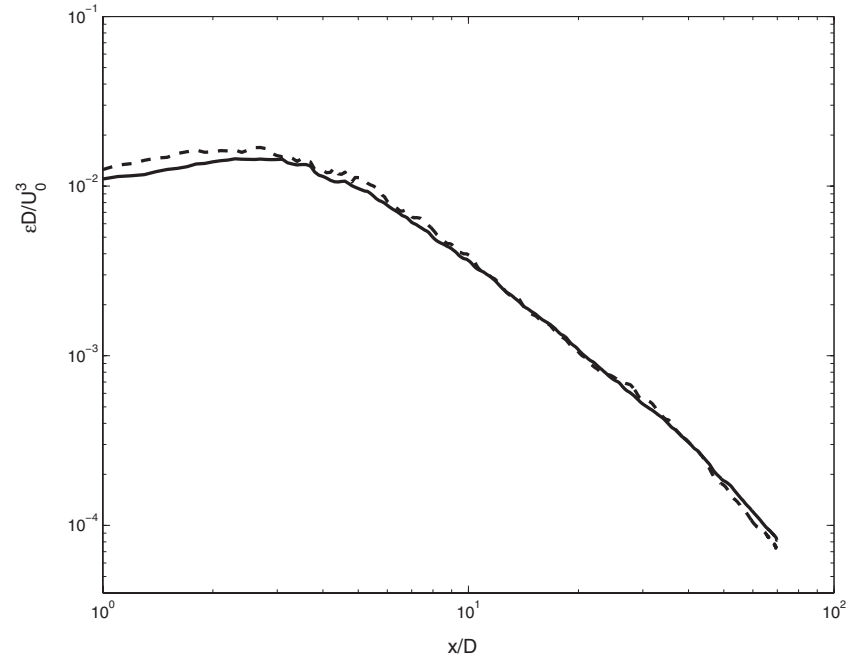

FIG. 16. Streamwise variation of the turbulent kinetic energy dissipation rate, $\epsilon$ along the crossbar centerline. Solid line: $\epsilon_{\text {true }}$, dashed line: $\epsilon_{i s o}$.

$$
\epsilon_{i s o}=15 \nu \overline{\left(\frac{\partial u}{\partial x}\right)^{2}},
$$

is also shown for comparison. There are particular features in the figure that need to be highlighted. First, and as expected, $\epsilon_{\text {true }}$ differs from $\epsilon_{i s o}$ for $x / D<10$, where the turbulence is far from being isotropic. However, as the turbulence decays, the two quantities tend to draw nearer, reflecting a trend of the turbulence to approach an approximate isotropic state. This is not too surprising. Indeed, behind the crossbar where the turbulence, which is being produced by the large scale motions, is highly nonisotropic, even at the dissipative scales. As the turbulence evolves along the streamwise direction, the large structures generate smaller scale motions. As the process continues, the small scale motions become dynamically separated from the large scale motions until they can be considered locally isotropic even if the flow is not globally isotropic. Since the dissipation is a small scales property, the isotropic estimate of the dissipation becomes more and more accurate as $x / D$ increases. Second, $\epsilon$ presents a clear change in its decay rate. The change occurs at $x / D$ of about 30 and is likely to be related to the inflexion point in the $q$ distribution, again pointing to a transition in the turbulence decay.

\section{CONCLUDING REMARKS}

The structure of the near-wake of a crossbar (made of two perpendicular square bars in a biplane arrangement) have been investigated through direct numerical simulations using the lattice Boltzmann method. Just behind the crossbar, the wake is made up of the individual wakes associated with each bar. The two wakes remain quite distinct for $x / D \leq 5$, except in the central region.

Fingerlike structures (regions of high longitudinal speed) are generated along the span of the bars and extend for about one to two bar diameters downstream. These fingerlike structures are associated with spanwise motions. It is argued that these latter are correlated with concentrations of streamwise vorticity along the bar span which mark the presence of streamwise vortical structures. It is observed that while the turbulent kinetic energy increases in the region $x / D \leq 5$, the contribution from its components varies. For example, for $x / D \leq 1$ the contribution from the streamwise component is larger than the contributions of the other two, but becomes the smallest for $x / D \geq 1$. The kinetic energy reaches its maximum at around $3 \leq x / D \leq 4$ and then decays. During this decay the relative contributions from the components of turbulent kinetic energy remain unchanged until about $x / d$ $=45$ where the streamwise component contribution becomes dominant once again.

Quite interestingly, the behavior observed in the centerline variations of the turbulent kinetic energy, its individual components and dissipation rate are similar to that observed in grid-generated turbulence. Of course a grid-generated turbulence is made up of the interactions of many crossbar wakes. Nevertheless, the present results suggest that one can expect to observe approximate homogeneous isotropic turbulence along the centerline of the mid- and far-wake of a crossbar. This can be used to isolate some of the parameters of the initial conditions in grid-generated turbulence. For instance, the focus can be placed on the shape of the bars and crossing arrangement, while the effect of the development and interactions of parallel wakes are not present. Clearly, in order to validate the present argument, a comparative study between a crossbar wake and grid-generated turbulence is required where the geometries of the bars used for the crossbar and the grid are identical.

\section{ACKNOWLEDGMENTS}

L.D. thanks Professor F. Anselmet and Dr. M. Amielh for their help with the PIV measurements carried out in their water tunnel facility at the Institut de Recherche sur les Phénomènes Hors Equilibre (IRPHE), Marseille.
[1] W. K. George, Phys. Fluids 4, 1492 (1992).

[2] W. K. George, H. Wang, C. Wollblad, and T. G. Johanson, in Proceedings of the 14th Australasian Fluid Mechanics Conference (AFMC), Adelaide, 10-14 December, 2001.

[3] P. Lavoie, P. Burattini, L. Djenidi, and R. A. Antonia, Exp. Fluids 39, 865 (2005).
[4] P. Lavoie, L. Djenidi, and R. A. Antonia, Book of Extended Abstract, Whither Turbulence Prediction and Control Conference, Seoul, Korea, March 26-29, 2006.

[5] P. Lavoie, Ph.D. thesis, University of Newcastle, Newcastle, Australia, 2006.

[6] G. Comte-Bellot and S. Corsin, J. Fluid Mech. 25, 657 (1966). 
[7] H. Osaka, I. Nakamura, H. Yamada, Y. Kuwata, and Y. Kageyama, Bull. JSME 26, 356 (1983).

[8] H. Osaka, H. Yamada, I. Nakamura, Y. Kuwata, and Y. Kageyama, Bull. JSME 26, 521 (1983).

[9] U. Frisch, B. Hasslacher, and Y. Pomeau, Phys. Rev. Lett. 56, 1505 (1986).

[10] S. Chen and G. D. Doolen, Annu. Rev. Fluid Mech. 30, 329 (1998).

[11] L. Djenidi, J. Fluid Mech. 552, 13 (2006).

[12] S. Hou, J. Sterling, S. Chen, and G. D. Doolen, Fields Inst.
Commun. 6, 151 (1996).

[13] D. K. McLaughlin and W. G. Tiederman, Phys. Fluids 16, 2082 (1973).

[14] M. Uberoi, J. Aeronaut. Sci. 23, 754 (1956).

[15] J. C. Lin, P. Vorobieff, and D. Rockwell, Phys. Fluids 8, 555 (1996).

[16] M. Braza, Flow Turbul. Combus. 63, 315 (2000).

[17] C. Williamson, Annu. Rev. Fluid Mech. 28, 477 (1996).

[18] Jayesh and Z. Warhaft, Phys. Fluids A 4, 2292 (1992). 